\title{
FDEIR: Content-Based Image Retrieval using Fast Demeanor Ensemble Features
}

\author{
Punit Soni ${ }^{1}$, Vijay Kumar Lamba ${ }^{2}$, Surender Kumar ${ }^{3}$ \\ ${ }^{1}$ Research Scholar, IKGPTU, Kapurthala, Punjab, India \\ ${ }^{2}$ Professor \& Principal, GCET, Khanpur Khui, Anandpur Sahib, Punjab, India \\ ${ }^{3}$ Associate Professor, GTB College, Bawanigarh, Punjab, India
}

Article History: Received: 10 November 2020; Revised: 12 January 2021; Accepted: 27 January 2021; Published online: 05 April 2021 Abstract: The demand for CBIR-based systems is increasing day-by-day with the increase of its applications.
Nowadays, various digital information systems like Medicine, Digital Libraries, Biodiversity information systems,
Fingerprint Identification, crime prevention, and many more are in trend, and all these systems need accuracy.
Several Automated Systems were earlier developed using features to improve the systems' accuracy, but it
introduces complexity and speed. A fast Demeanor Ensemble features-based approach is proposed to cope with
this issue, which also deals with accuracy, speeds up the system, and reduces its complexity. Different shape-based
features are used to form 3-set composite features, followed by the selection phase where the ALO algorithm is
utilized and reduces system complexity. The accuracy of the proposed system is estimated using distance-based
matching methods. Correl-1000 dataset is used to analyze the system's performance based on different metrics,
and the results show the proposed system's ability.

Keywords: CBIR, Ensemble Approach, Fast, Demeanor, Optimization, ALO.

\section{Introduction}

The advanced world of high modern technologies brings to large-scale information. Its keeping and processing need additional and more computational memory, storage space, graphical resources, and so on. A lot more than twenty years, the worldwide globe study community is dedicated to the difficulty of CBIR formulas' effectiveness and efficiency. Content-based image retrieval is widely relevant when looking at the areas of knowledge, medicine, culture, heritage, GIS, satellite images, structure, criminology, yet others [1]. In CBIR systems, the Content of an image is preferred over its metadata, and it is represented by a different set of shape, color, and texture-based features. Sometimes, the keyword was not worked effectively to produce image results, so content-based approaches define CBIR's success [2].

The CBIR system's performance is counted as useful if the system's retrieval rate is high, which is achieved only when the system returns the query samples' replica. CBIR systems belong to different research communities, mainly computer vision, image processing, database management, database mining, etc. [3]. The general architecture of the CBIR system follows the process of preprocessing, feature extraction, and matching. The first preprocessing step helps the system to clean the sample image and enhances its quality to extract features in the next phases. These features are visual and can be color, texture, spatial, region, or shape features. All features generate their own feature set and are used for matching purposes. Finally, the matching can be done using similarity-based measures in which distance-based methods are commonly used in the CBIR system [4]. Both features and similarity measures have their importance in the CBIR systems [5].

Several CBIR systems were earlier developed base on different measures and factors. For Instance, A Bi-layer CBIR [6] consist of two modules in which color, texture, and shape features were extracted in the first module and then the second phase; firstly, the matching was based on shape and texture and selected the subset of sample and finally matched with color and shape features to generate the output. The performance of this technique was impressive and analyzed from their experimentation. The other multi-trend structure descriptor CBIR encoded the color, edge, texture, and orientation information of equal or small or large size [7]. The approach was tested on correl dataset and achieved the best performance over the traditional approaches. The other multi-level featurebased approach is proposed for CBIR [8]. This proposed approach provides the binary pattern, magnitude, and other features using GLCM. Particle swarm optimization was used as a feature selector to reduce the system's complexity and improve its performance. The combinational approaches were also popular among others. The local phase quantization, ternary pattern, and wavelet base approach were proposed [9] to provide the best-matched results.

A semantic gap is a crucial issue in the existing CBIR systems that degrading the system's performance. SURF and HOG-based features [10], Shape adaptive DCT [11], clustering approach [12], spatial contents [13], and extensive, robust features [14] were proposed to resolve this issue and eliminates the semantic gaps between the 
query and the retrieved samples. These methods' performance was evaluated on different datasets by calculating different parameters and results in a superior version. It has been noticed from the above literature that some of the proposed systems uses combinational approaches that provides the best results, but the performance issue rises and increases the complexity of these CBIR systems.

The next section of this paper mainly discussed the feature extraction approaches in CBIR followed by optimization, and then deliberated the proposed demeanor approach and proposed CBIR model with experimentation and results.

\section{Feature Extraction Approaches}

Content-based Image Retrieval Systems are typically designed to retrieve a similar image from the database by comparing it with the query sample using different features. An image sample has quantitative and qualitative features that mainly comprise color, texture, shape, and semantics [15]. These features have a significant role in improving the CBIR system's performance and retrieving similar data productively. Some of the proposed feature extraction approaches developed for the existing CBIR systems are discussed in this section.

Mehmood et al. [16] proposed a novel feature-based approach in which they extracted the bag of visual features (BoVW) using a histogram. These BoVW extracted the keypoints and calculated the feature on its basis. In this work, the SIFT algorithm was used to extract the key points and generate the feature vector. The proposed approach shows valuable results based on different performance measures. Color and texture components also provide the relevant feature set. Fadaei et al. [17] proposed a color and texture-based approach using the Particle Swarm Optimization algorithm. They used wavelet and curvelet based approaches for feature extraction and combined them. The proposed algorithm achieved an average precision of 76.5\%. Some other features like color cooccurrence features, color histogram, and bit pattern features, were used by Admile and Shawan [18]. Hierarchicallocal-feature extraction [19] with three-level TREE Hierarchical data structure also shows its significant performance.

The combinational feature extraction approaches were introduced from the past few years, and it also provides effective results over the single features. Shape, color, and texture are the essential components of an image and will collectively provide efficient results. Various researchers proposed feature extraction approaches based on these components and emphasize their importance in the CBIR systems [20-28]. Color and texture have limited components, but the shape does not have any restrictions. So, numerous methods earlier proposed to extract shapebased features, SIFT, SURF, BRISK, HoG, LBP, and many more.

\section{Proposed Optimization Approach}

Optimization plays a vital role in order to improve the system's performance. Nowadays, different optimization approaches are added into many fields and show the effectiveness of their use. The optimization requirement is accelerated in the CBIR system due to the enlargement of the feature vector. The feature optimization approaches are of two types mainly, feature transform and feature selection. A feature selection approach selects the best possible features from the images to reduce the feature vector's size and reduces its complexity [29]. Though the selection contributed to the system's performance, the data loss must be the biggest question. So, selection must be the best, original, and crucial features in the reduced feature set. Filter and Wrapper based methods are nowadays commonly used for feature selection [30]. These days, metaheuristic approaches also showcase their effectiveness for both efficiency and speed. These approaches are wrapper based method and use supervised and unsupervised learning methods for the purpose of selection. Several approaches were earlier derived for the same purpose and hence revealed the strength of the optimization.

Belattar et al. [32] use a metaheuristic approach, a Genetic algorithm (GA). This approach works on the principle of genetics and natural selection. As per the studies, it is evaluated that sometimes composed offsprings are sometimes superior to the parents, and chromosomes for their survival follow the same concept. This work was proposed to select the skin lesion region from the samples, and this proposed approach improves the system's performance. Genetic algorithm is the most popular approach, and its number of variants were generated day-byday. The other instance of the GA was proposed by Chiesa et al. [35] to identify the robust set of features in highdimensional datasets. Diploid GA, another advanced interpretation, was proposed by Jasuja [36] to reduce the number of features. In this, genetics's dominance operator was used while creating a new population in GA, which enhances population quality means selects the best set of features. 
A hybrid variant of GA was proposed by Khotimah et al. [37] in which a simple probabilistic naïve based approach is mixed with GA. The capabilities of a naïve based approach to handle the complicated situation is very much acceptable, so it improves the proposed hybrid variant's performance in an adequate manner. The results also show the improvement rate of the proposed technique. The neoteric rendition using swarm optimized was proposed by Ding et al. [38] and tested using UC Irvine Machine learning repository to reduce the computational efficiency of the system. Different classification methods were also combined with GA to improve its performance in order to achieve the best results [39].

The other approach for selection is Binary Gravitation Search [BGS] and is proposed by Rashedi and pour [33]. This approach is a heuristic search algorithm and is designed to solve binary problems. They have used color, texture, and edge-based features in their work, and then the selection was performed. The performance in terms of precision was increased using the proposed method.

An advanced version of the optimization techniques is used nowadays in which machine learning and soft computing approaches are worked together to provide efficient solutions. Similarly, Kaur and Singh [34] proposed Cuckoo Search and Lion based optimization technique to select the features from the extensive feature set. They compared the optimizers' performance and analyzed that Lion based optimizer has a higher precision value than the Cuckoo method. The other soft computing approaches like Ant Colony Optimization [40], Forest Optimization [41], K-Lion Optimization [42], Ant-Lion Optimization [43], Whale Optimization [44,46], Binary Social Mimic Optimization [45] were also proposed to optimize the process of feature selection in any field of work.

\subsection{Proposed Demeanor Approach for Optimization}

A demeanor based optimization approach is proposed in this work that uses an Ant-Lion Optimization Algorithm (ALO). ALO is the multi-objective optimization that reduces complex problems and provides effective results. Recently, ALO was used to solve various engineering problems [47], scheduling issues [48], power systems [49], and sizing of resources problem [50] and performed well in each of the fields over the other traditional approaches.

ALO is a metaheuristic approach that follows antlions' foraging behavior with arbitrary or random walks to select the agents. This algorithm worked in two stages: (a) Build a trap, and (b) Catching prey and re-assembling the hole. In the first stage, the antlions' chasing capacity is demonstrated, for which a roulette wheel selection method is used. This method helps to administrate the antlions for wellness and distribution. The second stage represents that the insect goes inside the sand if it catches, and the new prey's recent positions are estimated. The whole process of ALO is a four-step process that starts with the sliding ants towards the antlion and then traps their holes. Finally, with the random walks of ants, elitism must be connected to obtain efficient results.

The pseudo-code of the proposed ALO algorithm for feature selection is as given below:

Affiliations. The affiliated institutions are to be listed directly below the names of the authors. Multiple affiliatons should be marked with superscript arabic numbers, and they should each start on a new line as shown in this document. In addition to the name of your affiliation, we would ask you to give the town and the country in which it is situated. Do not include the entire postal address. E-mail addresses should start on a new line and should be grouped per affiliation.

Headings. Headings should be capitalized (i.e., nouns, verbs, and all other words except articles, prepositions, and conjunctions should be set with an initial capital) and should, with the exception of the title, be aligned to the left. Only the first two levels of section headings should be numbered, as shown in Table 1. The respective font sizes are also given in Table 1 . Kindly refrain from using " 0 " when numbering your section headings.

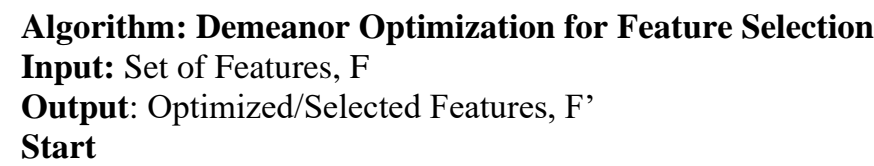

Step 1: The population of ant and antlions is generated and distributed over the sample image.

Step 2: Set position of the ant and antlions over the extracted feature vector of size $\mathrm{m} \mathrm{x} \mathrm{n}$.

Step 3: Compute Fitness for each ant and antlion

Step 4: Find the fittest antlion, and set Elite

Step 5: Intialize val=0 and while val <= size (sample)

Step 5.1: For each ant, (image pixel)

(a) Select antlion using Roulette wheel selection operator 
(b) Slide the ant to antlion and select the feature

(c) Generate an arbitrary walk for ant and normalize it for modeling End of For

Step 5.2: Re-evaluate the fitness for each ant

Step 5.3: if ant is fitter than antlion

Replace the antlion with its corresponding ant

End of if

Step 5.4: If antlion fitter than the current Elite Update Elite

End of while End of if

Stop

\section{Proposed Content-based Image Retrieval System}

This proposed system is designed to extract the relevant data from the system's information to achieve high accuracy. This model works similarly to the other Content-based Image retrieval; the difference is the FDEIR approach's inclusion. In this model, the work is divided into two phases:

(a) Training Phase in which all the data is prepared and stored,

(b) Testing phase that deals with the real-time query of the sample.

The training data is prepared using the dataset of features in which Demeanor Ensemble features are collected. Here, the features are 3 -set composite shape features and are different combinations of SIFT, SURF, BRISK, HoG, FAST, and PCA-SIFT. The extracted ensemble features are very high, making the system slow and complicated. So, to reduce the number of features and select the optimal feature values, an Ant Lion Optimization algorithm is used in this work. The detailed process of this proposed work is shown in the figure given below. The figure deliberates the CBIR system's process to retrieve the relevant image data from the database. In this work, the top 10 matches are fetched from the existed database if matched with the query sample. The proposed model generally performs the following steps for its implementation and generalization. These steps are:

Data Acquisition and Pre-processing: The first step for both training and query samples is to acquire them and then remove the unwanted noise to improve its quality. The samples' quality is enhanced using the 2-Dimensional filtration approach. The filter generates the mask of different sizes and helps to discard the undesirable pixel values. It improves the quality of the image sample and enhances its features for easy detection and extraction.

Ensemble Feature Extraction: Feature extraction is an essential step for the CBIR system and plays a significant role in processing the samples. In image processing, features are categorized into color-based, shape-based, and texture-based. All these features provide information on the sample images. For this work, shape-based features are selected with 3-set composite features. In this 3-set composite, different feature extraction methods are combined and then form an ensemble feature vector. For analysis, BRISK, FAST, HoG, SIFT, SURF, and PCA-SIFT algorithms are used, and six different Ensemble vectors are generated using diverse combinations.

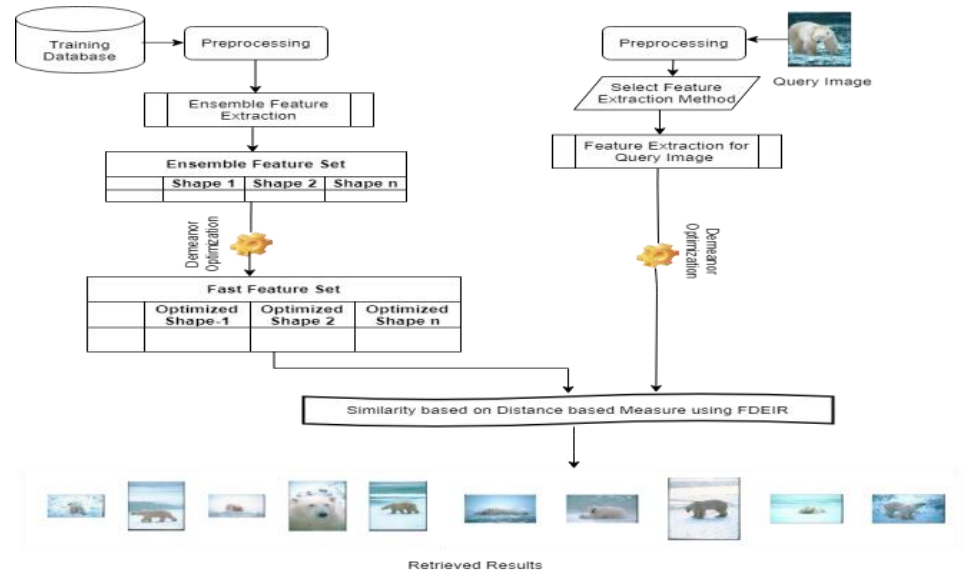

Figure 1. Fast Demeanor Ensemble features-based CBIR 
Ensemble features are the set of fully composite features, and it is defined as a set of features generated using a Union component of the feature set. fig. 2 shows the set of features F1, F2, and F3 to create a combined vector to form a 3 -set composite feature vector and is calculated as:

$$
C_{f}=F 1\left[\begin{array}{cccc}
f_{11} & f_{12} & \ldots \ldots & f_{n 1} \\
f_{21} & f_{22} & \ldots \ldots & f_{n 2} \\
\ldots & \ldots & \ldots \ldots & \ldots \\
f_{m 1} & f_{m 2} & \ldots \ldots & f_{m n}
\end{array}\right] \cup\left\{F 2\left[\begin{array}{cccc}
f_{11} & f_{12} & \ldots \ldots & f_{n 1} \\
f_{21} & f_{22} & \ldots \ldots & f_{n 2} \\
\ldots & \ldots & \ldots \ldots & \ldots \\
f_{m 1} & f_{m 2} & \ldots \ldots & f_{m n}
\end{array}\right] \cup F 3\left[\begin{array}{cccc}
f_{11} & f_{12} & \ldots \ldots & f_{n 1} \\
f_{21} & f_{22} & \ldots \ldots & f_{n 2} \\
\ldots & \ldots & \ldots \ldots & \ldots \\
f_{m 1} & f_{m 2} & \ldots \ldots & f_{m n}
\end{array}\right]\right\}
$$

Where, $C_{f}$ represents the composite feature vector. In this work, 6-different sets of ensemble features are generated, and each has its different combinations shown in fig. 2. The combinations generate the ensemble feature set that improves the system's performance but raises complexity issues.

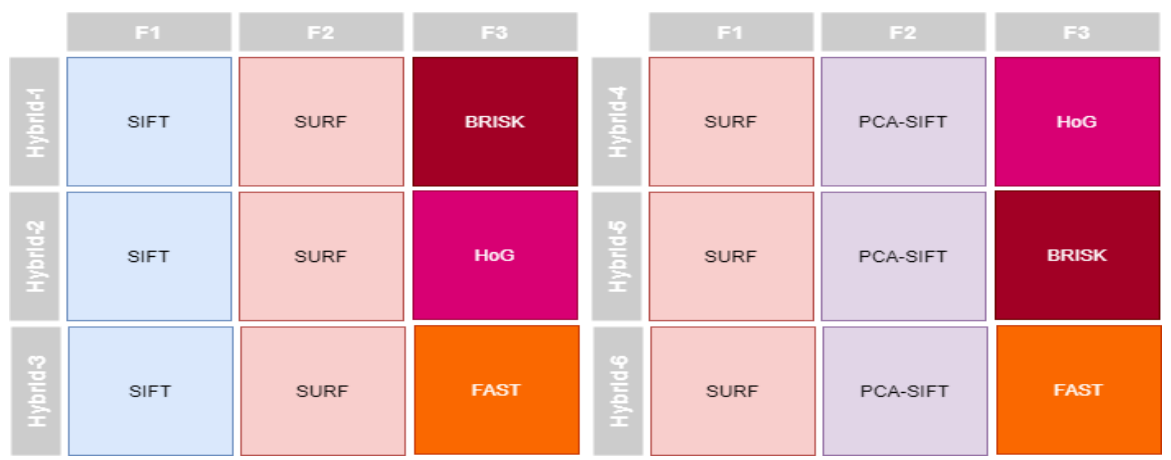

Figure 2. Set composite feature set (Ensemble Features)

Feature Optimization: It is not an essential step for every CBIR system, but in this work, due to ensembled features, complexity increases gradually and can cause performance issues. So, these issues can be handled with the help of the optimization method. Here in this work, the Demeanor-based optimization approach is used, which comprises the Antlion Optimization method. The detailed process is discussed in the previous section.

Matching and Retrieval: The final step of the proposed system is the retrieval of the data from the database that can be based on any similarity measure methods. In this work, Euclidean distance and the Hausdroff distance-based methods are used for similarity calculation and extraction of the data.

\section{Experimentation and Results}

The proposed FDEIR is implementing using a MATLAB simulator where the testing is performed using the Corel-1000 [51] dataset. This dataset contains 10000 images of 100 semantic classes, where each class contains 100 images. In this work, the system is trained using 5000 samples of 100 semantic classes, and each class contains 50 images. The testing can be performed using any query sample image, and matching is retrieved from the trained 5000 samples. The proposed work's performance is analyzed based on different parameters, namely, Number of Features, Feature Extraction Time, Feature Selection Time, and Accuracy. The details of these parameters and their results are as given below:

(a) Number of Features: Features play a significant role in finding out the object from the image. So, here different shape-based features and also the ensemble features up to 3 -set are extracted. The extracted numbers of features for some of the image samples are as given in table 1 and table 2.

The tables provides the details of the number of features of some samples for single as well as ensemble feature extraction techniques. Here for the first three hybrid combinations (i.e., Hybrid-1 to 3), SIFT and SURF combined with BRISK, HoG, and FAST, respectively, and for the next combinations (i.e., Hybrid4 to 6), SURF and PCA-SIFT are combined with HoG, BRISK and FAST respectively.

Table 1. Number of Features

\begin{tabular}{|c|c|c|c|c|c|c|}
\hline Samples & Brisk & Fast & HoG & Sift & SURF & PCA-SIFT \\
\hline Image-1 & 14016 & 239 & 11088 & 85248 & 6784 & 19721 \\
\hline Image-2 & 11776 & 208 & 11088 & 57984 & 5888 & 23749 \\
\hline
\end{tabular}




\begin{tabular}{|c|c|c|c|c|c|c|}
\hline Image-3 & 10880 & 172 & 11088 & 115072 & 5824 & 15169 \\
\hline Image-4 & 10176 & 176 & 11088 & 61824 & 6976 & 15504 \\
\hline Image-5 & 10368 & 177 & 11088 & 61184 & 7488 & 19930 \\
\hline Image-6 & 960 & 17 & 11088 & 4224 & 2176 & 10647 \\
\hline Image-7 & 1344 & 19 & 11088 & 127488 & 1408 & 8959 \\
\hline Image-8 & 768 & 10 & 11088 & 5120 & 2240 & 9466 \\
\hline Image-9 & 2048 & 42 & 11088 & 30720 & 3904 & 8276 \\
\hline Image-10 & 2176 & 43 & 11088 & 20352 & 4032 & 11692 \\
\hline
\end{tabular}

Table 2. Number of Features (contd.)

\begin{tabular}{|c|c|c|c|c|c|c|}
\hline Samples & Hybrid-1 & Hybrid-2 & Hybrid-3 & Hybrid-4 & Hybrid-5 & Hybrid-6 \\
\hline Image-1 & 106048 & 103120 & 92271 & 57652 & 60580 & 46564 \\
\hline Image-2 & 75648 & 74960 & 64080 & 64156 & 64844 & 53068 \\
\hline Image-3 & 131776 & 131984 & 121068 & 47172 & 46964 & 36084 \\
\hline Image-4 & 78976 & 79888 & 68976 & 55044 & 54132 & 43956 \\
\hline Image-5 & 79040 & 79760 & 68849 & 58956 & 58236 & 47868 \\
\hline Image-6 & 7360 & 17488 & 6417 & 36004 & 25876 & 24916 \\
\hline Image-7 & 130240 & 139984 & 128915 & 30876 & 21132 & 19788 \\
\hline Image-8 & 8128 & 18448 & 7370 & 32008 & 21688 & 20920 \\
\hline Image-9 & 36672 & 45712 & 34666 & 30992 & 21952 & 19904 \\
\hline Image-10 & 26560 & 35472 & 24427 & 38320 & 29408 & 27232 \\
\hline
\end{tabular}

It is clear from the results that the number of features is enormous in number for hybrid combinations than single feature extraction methods. Though the performance will be improved using the large set of features, the complexity increases. So, to balance this and reduce the complexity, these extracted features are optimized using the Demeanor approach, which reduces the number of features and selects only the optimal features. Fig 3 portrays the average results of the feature extraction with and without the optimization technique.

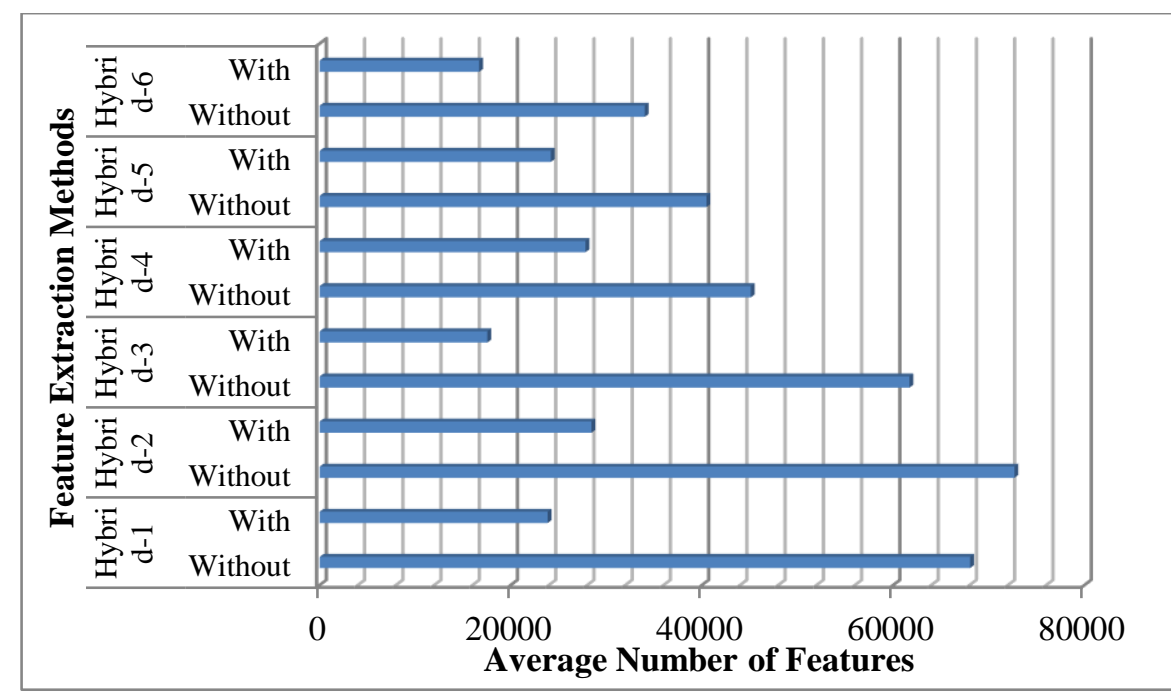

Figure 3. Average Number of Features (With and Without Optimization)

The above figure describes the optimization results over the hybrid feature extraction algorithms and details the number of features reduced or optimized by using the Demeanor-based approach. It is clear from the results that the optimization reduces the number of features without affecting the features and provides effective results. The maximum number of reduced features from the above hybrid combination $\mathrm{s}$, i.e., $71.5 \%$, is the hybrid-3 algorithm, a combination of SIFT, SURF, and FAST. The feature optimization rate for all the hybrid methods is as given in figure 4. 


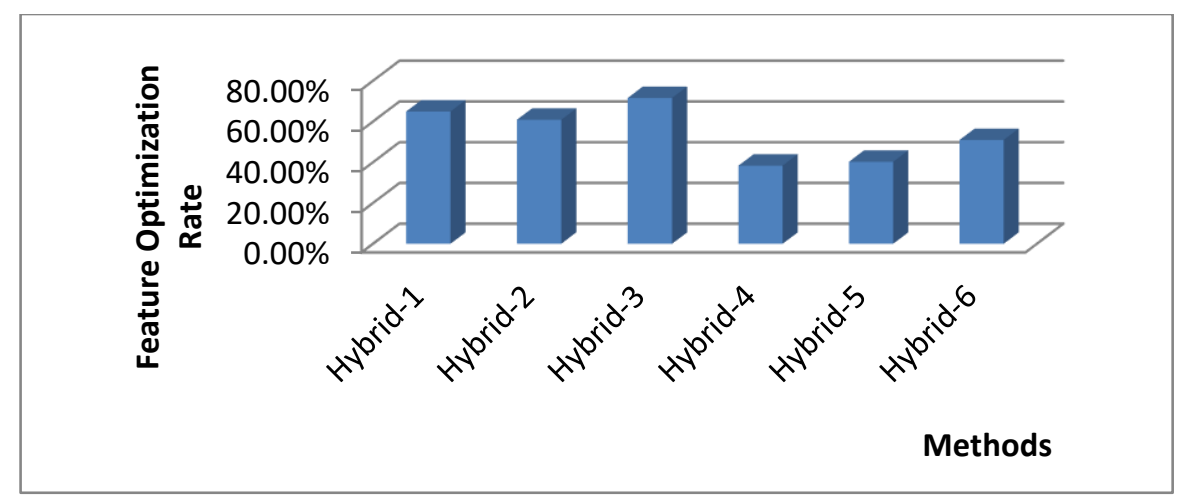

Figure 4. Feature Optimization Rate

(b) Feature Extraction Time: Time is an essential factor and dramatically influences the performance of the recognition system. The system performance is dependent on the speed, which depends on the time for computations so, the lesser the speed, the faster the system. The time for feature extraction is calculated for each method and is as shown in the table below:

Table 2. Average Time for Feature Extraction

\begin{tabular}{cccc}
$\begin{array}{c}\text { Feature } \\
\text { Extraction } \\
\text { Method }\end{array}$ & $\begin{array}{c}\text { Average Time for } \\
\text { Feature Extraction }\end{array}$ & $\begin{array}{c}\text { Feature } \\
\text { Extraction } \\
\text { Method }\end{array}$ & $\begin{array}{c}\text { Average Time for } \\
\text { Feature } \\
\text { Extraction }\end{array}$ \\
\hline Brisk & 0.37715166 & Hybrid-1 & 1.091528 \\
Fast & 0.02529262 & Hybrid-2 & 0.729979 \\
HOG & 0.03909294 & Hybrid-3 & 0.711011 \\
Sift & 0.86143923 & Hybrid-4 & 51.15882 \\
SURF & 0.03388625 & Hybrid-5 & 51.47945 \\
PCA-SIFT & 49.82400498 & Hybrid-6 & 52.70056 \\
\hline
\end{tabular}

The above results depict that the performance of hybrid- 3 is better in terms of feature extraction time than other composite features.

(c) Feature Selection Time: In this work, an optimization algorithm is added to select the optimal features to improve the system's performance. The time taken to select features can also affect the system's performance so, it should be less. The average feature selection time for different algorithms is as shown in the figure below:

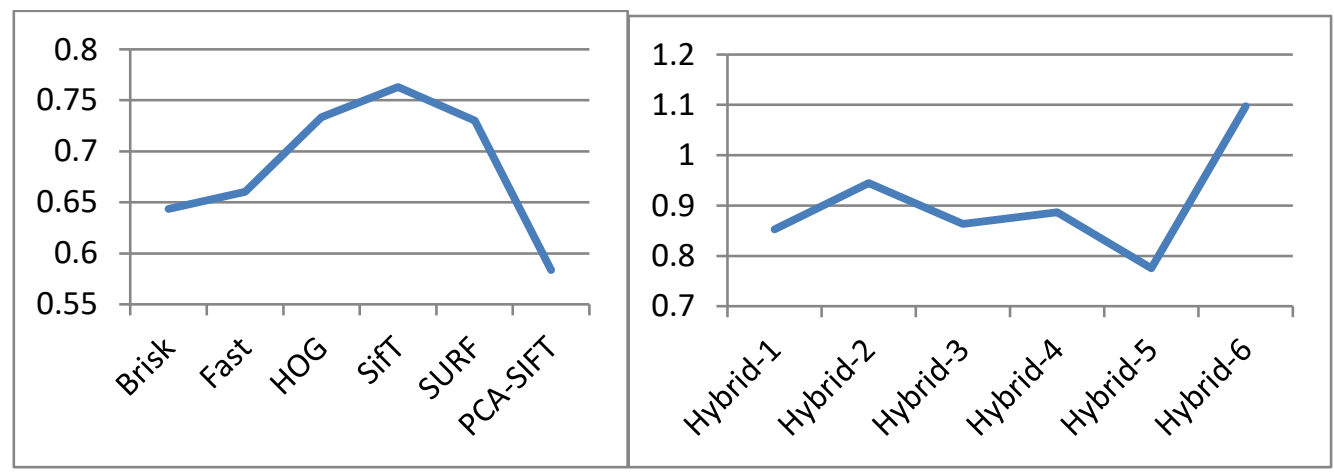

Figure 5. Feature Selection Time (a) Single, (b) Composite Method

The above results depicted that in single algorithms, PCA-SIFT took significantly less time for optimization, and in composite algorithms, Hybrid-5 has a lesser time than the other methods.

(d) Accuracy: Accuracy is an essential factor that helps measure the proposed system's performance and is calculated using two different techniques while retrieving samples from a large dataset. Table 3 shows the performance of the proposed system with different features and different methods. 
Table 3. Accuracy using Dataset Corel [19]

\begin{tabular}{cccccc}
$\begin{array}{c}\text { Feature } \\
\text { Extraction } \\
\text { Method }\end{array}$ & $\begin{array}{c}\text { Euclidean } \\
\text { Distance }\end{array}$ & $\begin{array}{c}\text { Hausdrauff } \\
\text { Distance }\end{array}$ & $\begin{array}{c}\text { Feature } \\
\text { Extraction } \\
\text { Method }\end{array}$ & $\begin{array}{c}\text { Euclidean } \\
\text { Distance }\end{array}$ & $\begin{array}{c}\text { Hausdrauff } \\
\text { Distance }\end{array}$ \\
\hline Brisk & $79.37 \%$ & $81.04 \%$ & Hybrid-1 & $85.64 \%$ & $87.54 \%$ \\
Fast & $\mathbf{8 0 . 2 0 \%}$ & $\mathbf{8 2 . 6 5 \%}$ & Hybrid-2 & $81.90 \%$ & $84.20 \%$ \\
HOG & $78.60 \%$ & $80.02 \%$ & Hybrid-3 & $\mathbf{8 9 . 4 5 \%}$ & $\mathbf{9 3 . 5 1 \%}$ \\
Sift & $75.10 \%$ & $77.90 \%$ & Hybrid-4 & $82.42 \%$ & $83.92 \%$ \\
SURF & $77.30 \%$ & $79.80 \%$ & Hybrid-5 & $83.87 \%$ & $86.51 \%$ \\
\hline PCA-SIFT & $72.65 \%$ & $76.58 \%$ & Hybrid-6 & $80.73 \%$ & $83.76 \%$ \\
\hline
\end{tabular}

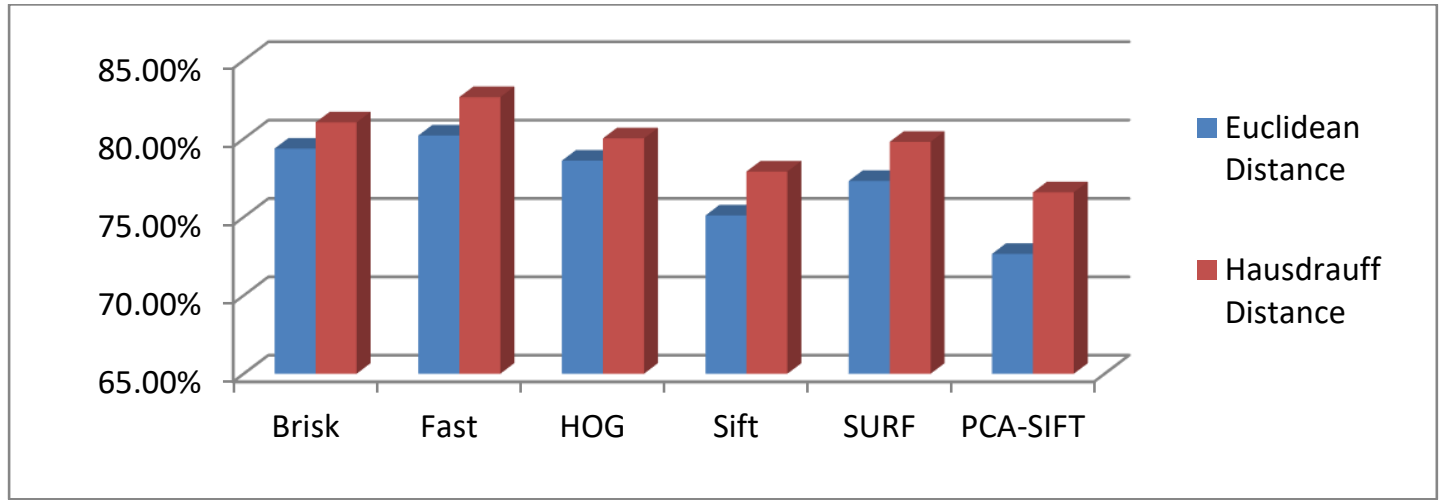

(a)

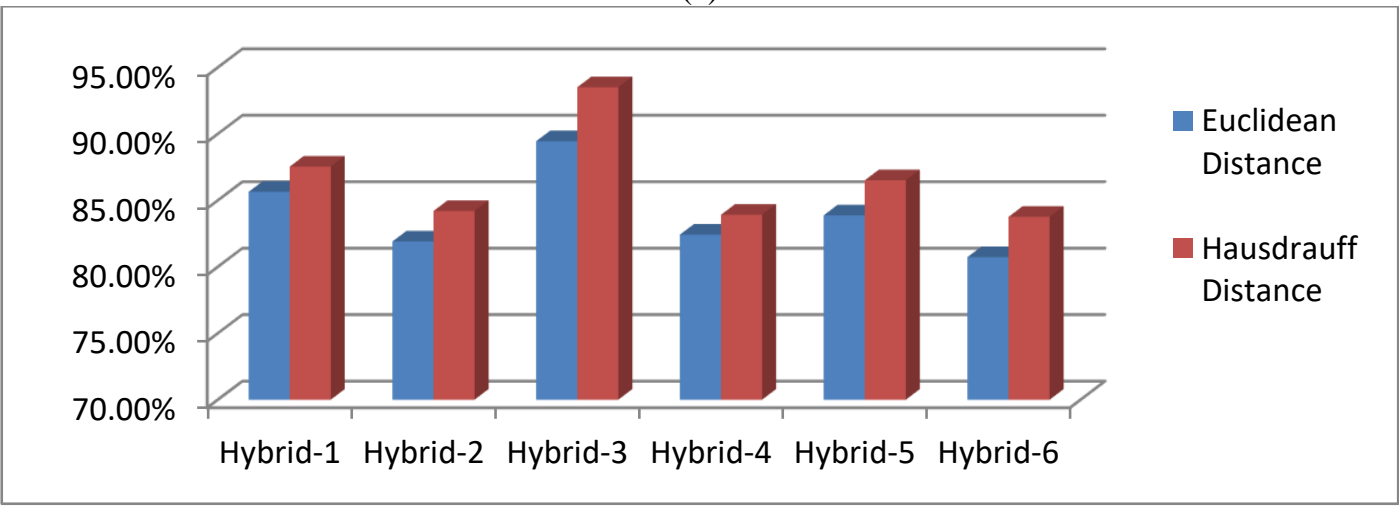

(b)

Figure 6. Accuracy of Different Feature Extraction Methods

The above results depict that the FAST algorithm's accuracy is better in single algorithms, whereas Hybrid-3 has the highest accuracy from all other feature extraction methods for both distance-based matching methods. In terms of the matching method, the Hausdrauff distance performance is better than the Euclidean distance and achieved an accuracy of $93.51 \%$.

\section{Conclusion}

The proposed system boosts the aspects of a content-based image retrieval system by its performance improvement. This system worked on a feature-based approach where different shape-based features are extracted, and then a 3-set composite feature set was formed. Optimization of the feature is another benefit provided by this system that is based on the demeanor approach. This contemporary approach not only selects the best features but also enhances its speed. The performance of the proposed approach is calculated using different parameters, and it is clear from the results that the accuracy of the system is better with the demeanor ensemble features compared to the single feature vectors for all combinations; also, in FDEIR, the Hybrid-3 approach's best performance using both Euclidean and Hausdrauff distance-based matching. Though the accuracy using the hausdrauff method is $4 \%$ better than the Euclidean distance, the matching time is its drawback. In the future, this work could be extended to reduce the matching time to enhance the CBIR systems' performance. 


\section{References}

1. Vetova, S., Draganov, I.: CBIR Efficiency Enhancement using Local Features Algorithm with Hausdorff Distance. Wseas Transactions on Computer Research, 5, 116-123 (2017)

2. Sharma, M., Batra, A.: Analysis of Distance Measures in Content Based Image Retrieval. Global Journal of Computer Science and Technology: G Interdisciplinary, 14(2), 1-7 (2014)

3. Yadav, O., Suryawanshi, V.: CBIR Evaluation using Different Distances and DWT. International Journal of Computer Applications, 93(16), 36-40 (2014)

4. Patil, S., Talbar, S.: Content Based Image Retrieval Using Various Distance Metrics. International Conference on Data Engineering and Management, 154-161 (2010)

5. M O, D., E R, V.: Performance Analysis of Distance Metric for Content Based Image Retrieval. International Journal of Engineering and Advanced Technology (IJEAT), 8(6), 2215-2218 (2019)

6. Singh, S., Batra, S.: An efficient bi-layer content based image retrieval system. Multimed Tools Appl 79, 17731-17759 (2020)

7. Sathiamoorthy, S., Natarajan, M.: An efficient Content based image retrieval using enhanced multitrend structure descriptor. SN Appl. Sci. 2, 217 (2020)

8. Garg, M., Dhiman, G.: A novel content-based image retrieval approach for classification using GLCM features and texture fused LBP variants. Neural Comput\&Applic (2020)

9. Kashif, M., Raja, G. \& Shaukat, F.: An Efficient Content-Based Image Retrieval System for the Diagnosis of Lung Diseases. J Digit Imaging 33, 971-987 (2020)

10. Mehmood, Z., Abbas, F., Mahmood, T. et al.: Content-Based Image Retrieval Based on Visual Words Fusion Versus Features Fusion of Local and Global Features. Arab J Sci Eng 43, 7265-7284 (2018)

11. Belalia, A., Belloulata, K. Kpalma, K.: Region-based image retrieval in the compressed domain using shape-adaptive DCT. Multimed Tools Appl 75, 10175-10199 (2016)

12. Pandey, S., \& Khanna, P.: Content-based image retrieval embedded with agglomerative clustering built on information loss. Computers \& Electrical Engineering, 54, 506-521 (2016)

13. Mehmood, Z., Mahmood, T. \& Javid, M. A.: Content-based image retrieval and semantic automatic image annotation based on the weighted average of triangular histograms using support vector machine. ApplIntell 48, 166-181 (2018)

14. Alsmadi, K, M.: An efficient similarity measure for Content based image retrieval using memetic algorithm,Egyptian Journal of Basic and Applied Sciences, 4,112-122 (2017)

15. Banerjee I, Kurtz C, Devorah AE, Do B, Rubin DL, Beaulieu CF.: Relevance feedback for enhancing Content based image retrieval and automatic prediction of semantic image features: Application to bone tumor radiographs. J Biomed Inform. Aug; 84:123-135 (2018)

16. Mehmood, Z., Anwa, S., \& Altaf, M.: A novel image retrieval based on rectangular spatial histograms of visual words. Kuwait Journal Science, 45(1), 54-69 (2018)

17. Fadaei, Sadegh\&Amirfattahi, Rassoul, Ahmadzadeh, M.R..: A New Content-Based Image Retrieval System Based on Optimized Integration of DCD, Wavelet and Curvelet Features. IET Image Processing. 11. 10.1049/iet-ipr.2016.0542 (2016)

18. S.Admile, N., R.Dhawan, R.: Content Based Image Retrieval Using Feature Extracted From Dot diffusion Block Truncation Coding. International Conference on Communication and Electronics Systems (ICCES), 1-6 (2016)

19. Jian, M., Yin, Y., Dong, J. et al.: Content-based image retrieval via a hierarchical-local-feature extraction scheme. Multimed Tools Appl 77, 29099-29117 (2018).

20. Bulli Babu, R., Vanitha, V., Sai Anish, K.: Content based Image Retrieval using Color, Texture, Shape and Active Re-Ranking Method. Indian Journal of Science and Technology, 9(17), 1-6 (2016)

21. Yue, J., Li, Z., Liub, L., Fub, Z.: Content-based image retrieval using color and texture fused features. Mathematical and Computer Modelling, 54, 1-7 (2011)

22. Pavithra, L., Sharmila, T.: An efficient framework for image retrieval using color, texture and edge features. Computers and Electrical Engineering, 70, 580-593 (2018)

23. Sotoodeh, M., Reza Moosavi, M., \& Boostani, R.: A novel adaptive LBP-based descriptor for color image retrieval. Expert Systems With Applications, 127, 342-352 (2019)

24. Dhanoa, J., Garg, A.: A Novel Technique for Shape Feature Extraction Using Content Based Image Retrieval. MATEC Web of Conferences, 57, 1-6 (2016)

25. Chaudhari, R., Patil, A.: Content Based Image Retrieval Using Color and Shape Features. International Journal of Advanced Research in Electrical, Electronics and Instrumentation Engineering, 1(5), 386-392 (2012)

26. Helta Dais, M., TamilSelv, D., GinuMol, J.: Combined texture and Shape Features for Content Based Image Retrieval. International Conference on Circuits, Power and Computing Technologies 
[ICCPCT-2013], 1-5 (2013)

27. Shirazi, S., Amin Khan, N., Umar, A., Razzak, M., Naz, S., AlHaqbani, B.: Content-Based Image Retrieval Using Texture Color Shape and Region. (IJACSA) International Journal of Advanced Computer Science and Applications, 7(1), 418-426 (2016)

28. Rehman, M., Sharıf, M., Raza, M.: Shape Features Extraction Method for Content based Image Retrieval. Sindh Unıversity Research Journal (Science Serıes), 48(1), 45-48 (2016)

29. Benloucif, S., Boucheham, B.: Impact of Feature Selection on the Performance of Content-Based Image Retrieval (CBIR). 4th International Symposium ISKO-Maghreb: Concepts and Tools for Knowledge Management (ISKO-Maghreb), 1-7 (2015)

30. Sarrafzadeh, A., Agh Atabay, H., Pedram, M., \& Shanbehzadeh, J.: ReliefF Based Feature Selection In Content-Based Image Retrieval. Proceedings of the International MultiConference of Engineers and Computer Scientists, 1-4 (2012)

31. Gurusamy, V., Nandhini, K.: Content Based Image Retrieval using Feature Selection Techniques. IJSRD - International Journal for Scientific Research \& Development, 5(5), 283-286 (2017)

32. Belatta, K., Mostefai, S., Draa, A.: A Hybrid GA-LDA Scheme for Feature Selection in ContentBased Image Retrieval. International Journal of Applied Metaheuristic Computing, 9(2), 48-71 (2018)

33. Rashedi, E., Nezamabadi-pour, H.: Improving the Precision of CBIR Systems by Feature Selection Using Binary Gravitational Search Algorithm. The 16th CSI International Symposium on Artificial Intelligence and Signal Processing (AISP 2012), 39-42 (2012)

34. Kaur, P., Kumar Singh, R.: Content-Based Image Retrieval Using Machine Learning And Soft Computing Techniques. International Journal Of Scientıfic \& Technology Research, 9(1), 1327$1332(2020)$

35. Chiesa, M., Maioli, G., Colombo, G., Piacentini, L.: GARS: Genetic Algorithm for the identification of a Robust Subset of features in high-dimensional datasets. BMC Bioinformatics, 1-11 (2020)

36. Jasuja, A.: Feature Selection Using Diploid Genetic Algorithm. Annals of Data Science, 1-11 (2019)

37. Khotimah, B. K., Miswanto, M., \& Suprajitno, H.: Optimization of Feature Selection Using Genetic Algorithm in Naïve Bayes Classification for Incomplete Data. International Journal of Intelligent Engineering and Systems, 13(1), 334-343 (2020)

38. Ding, Y., Zhou, K., \& Bi, W.: Feature selection based on hybridization of genetic algorithm and competitive swarm optimizer. Soft Computing, 1-10 (2020)

39. Garc1'a-Dominguez, A., Galva'n-Tejada, C., Zanella-Calzada, L., Gamboa-Rosales, H., Galva'nTejada, J., Celaya-Padilla, J., Magallanes-Quintanar, R.: Feature Selection Using Genetic Algorithms for the Generation of a Recognition and Classification of Children Activities Model Using Environmental Sound. Hindawi Mobile Information Systems, 1-12 (2020)

40. Neagoe, V., Neghina, E.: Feature Selection with Ant Colony Optimization and Its Applications for Pattern Recognition in Space Imagery. International Conference on Communications (COMM), 101-104 (2016)

41. Xie, Q., Cheng, G., Zhang, X., Lei, P.: Feature Selection Using Improved Forest Optimization Algorithm. Information Technology and Control, 49(2), 289-301 (2020)

42. G, J., Brunda, S.: An Improved K-Lion Optimization Algorithm With Feature Selection Methods for Text Document Cluster. International Journal of Computer Sciences and Engineering, 245-251 (2018)

43. Wang, M., Wu, C., Wang, L., Xiang, D., Huang, X.: A feature selection approach for hyperspectral image based on modified ant lion optimizer. Knowledge-Based Systems, 168, 39-48 (2019)

44. Mafarja, M., Mirjalili, S.: Whale Optimization Approaches for Wrapper Feature Selection. Applied Soft Computing, 1-32 (2017)

45. Kant 1 Ghosh, K., Singh, P. K., Hong, J., Geem, Z. W., Sarkar, R.: Binary Social Mimic Optimization Algorithm With X-Shaped Transfer Function for Feature Selection. IEEE Access, 8, 97890-97906 (2020)

46. Zawbaa, H., Emary, E., PARV, B.: Feature Selection Based on Antlion Optimization Algorithm. Third World Conference on Complex Systems (WCCS), 1-7 (2015)

47. [47] Mirjalili, S., Jangir, P., \& Saremi, S. (2016). Multi-objective ant lion optimizer: A multiobjective optimization algorithm for solving engineering problems. Applied Intelligence, 46(1), 7995. doi:10.1007/s10489-016-0825-8

48. Petrović, M., Petronijević, J., Mitıć, M., Vuković, N., Miljković, Z., Babıć, B.: The Ant Lion Optimization Algorithm for Integrated Process Planning and Scheduling. Applied Mechanics and Materials, 834, 187-192 (2016)

49. Mouassa, S., Bouktir, T., Salhi b, A.: Ant lion optimizer for solving optimal reactive power dispatch problem in power systems. Engineering Science and Technology, an International Journal, 1-11 
(2017)

50. Reddy, D. P., Reddy, V., Manohar T, G.: Ant Lion optimization algorithm for optimal sizing of renewable energy resources for loss reduction in distribution systems. Journal of Electrical Systems and Information Technology, 5(3), 663-680 (2018)

51. Wang, J., Li, J., Wiederhold, G.: SIMPLIcity: Semantics-Sensitive Integrated Matching for Picture Libraries. IEEE Transactions on Pattern Analysis and Machine Intelligence, 23(9), pp.947-963 (2001) 